\title{
Implante Auditivo de Tronco Cerebral en el tratamiento de la hipoacusia sensorio neural
}

\author{
Auditory Brainstem Implant in the treatment \\ of sensoneural hypoacousia
}

Jorge Caro $L^{1}$, Jimena Cevo $E^{2}$.

\begin{abstract}
RESUMEN
Los Implantes Auditivos de Tronco Cerebral (IATC) estimulan directamenteel núcleo coclear en el tronco encefálico y fueron creados para las personas con neurofibromatosis tipo 2 (NF2). La correcta colocación del IATC es comprobada en forma intraoperatoria con los potenciales evocados de troncoencéfalo (EABR). desempeño observado con el IATC no es tan bueno como el obtenido con un implante coclear, pero se ha observado que sirven como ayuda para la lectura de labios. Los primeros resultados son promisorios e incluye, en algunos de los pacientes implantados, cierta habilidad en las pruebas de formato abierto. Actualmente se continúa investigando para mejorar su desempeño.

Palabras clave: Implante Auditivo de Tronco Cerebral, neurofibromatosis tipo 2, hipoacusia sensorioneural.
\end{abstract}

\section{SUMMARY}

Auditory Brainstem Implants (IATC) directly stimulate the cochlear nucleus in the encephalic stem and were created for patients suffering from neurofibramotosis type 2 (NF2). The correct placement of the IATC is verified intraoperatively through the response to the tests on the brain stem (EABR). The performance with IATC is not as good as with the Cochlear Implant, but it has been noticed that they do help with lip reading. The first results are promising and include a certain speech ability in the open format tests in some of the implanted patients. More investigations are being conducted in order to improve its performance.

Kay words: Auditory Brainstem Implant, Neurofibromatosis type 2, sensoneural hipoacousia.

\footnotetext{
${ }^{1}$ Unidad Docente Académica de Otorrinolaringología, Hospital Gínico Pontificia Universidad Católica de Chile.

2 Interna Escuela de Medicina, Pontificia Universidad Católica de Chile
} 


\section{INTRODUCCIÓN}

La hipoacusia en sus distintos grados es ampliamente reconocida como uno de los desórdenes más comunes de la humanidad, afecta aproximadamente a 100 millones de personas alrededor del mundo ${ }^{1}$, sólo en Estados Unidos existen más de 28 millones de americanos que la padecen ${ }^{2}$ y se espera que estas cifras se incrementen significativamente durante las próximas décadas.

Se ha reportado que 5 de cada 10.000 niños menores de 2 años padece de hipoacusia severa? Si bien el problema es crítico para los adultos es dramático para los niños, en quienes la hipoacusia no puede ser vista como un déficit aislado, ya que se asocia a varias restricciones psicosociales al producir devastadoras consecuencias en el desarrollo del lenguaje y en su aprendizaje disminuyendo su interacción con el medio que los rodea.

La hipoacusia sensorioneural (HSN) es causada por un defecto en el oído interno o en la vías auditivas centrales. Su tratamiento depende del grado de hipoacusia; para las HSN moderadas a severas se utilizan audífonos, mientras que en aquellos casos de hipoacusias profundas o severas por defecto coclear con un nervio auditivo intacto se puede utilizar un implante coclear ${ }^{2}$. Desgraciadamente la colocación de estos implantes no siempre es posible, por esto se han desarrollado los implantes auditivos de tronco cerebral (IATC). En 1979 Hitselberger y col fueron los primeros en implantar un par de electrodos en el núcleo coclear, en 1992 Lazig y col iniciaron un estudio piloto para desarrollar un IATC multicanal de 20 electrodos, el diseño final fue el dispositivo de 21 canales usado hasta 1999 , cuando se introdujo nucleus $24 \mathrm{ABI}^{2}$.

En esta revisión tratamos de entregar una visión general acerca de qué son los implantes de tronco cerebral, su mecanismo de acción y los resultados que se han obtenido con éstos en los distintos centros que realizan este tipo de cirugía.

\section{¿QUÉ ES UN IMPLANTE DE TRONCO CEREBRAL?}

日 IATC es un dispositivo que provee percepción del sonido a través de electrodos implantados quirúrgicamente. Funciona de manera similar al implante coclear pero está creado para omitir la cóclea y el nervio coclear estimulando directamente el núcleo coclear en el troncoencéfalo ${ }^{3}$. Es decir, estimula a las neuronas de segundo orden.

Contiene: un equipo receptor/estimulador, un portaelectrodos de silicona $(3 \times 8,5 \mathrm{~mm})$ con 21 electrodos de platino dispuestos en 3 filas (diámetro electrodo $0,7 \mathrm{~mm}$ ), un dispositivo de Dacron que estabiliza los electrodos al permitir el crecimiento de tejido conectivo alrededor de éste y un imán del receptor que es removible 4 (Fgura 1).

日 sonido es recibido por el micrófono colocado detrás del pabellón auricular, luego es transmitido al procesador del lenguaje, que transforma la información acústica en digital en otras palabras los sonidos son codificados, luego estas señales

Figura 1. Implante Auditivo de Tronco Cerebral. 
son transmitidas al receptor interno y de éste hacia los electrodos, que estimulan el núcleo auditivo. Desde donde se transmite la información al cerebro para ser interpretada (Figura 2).

\section{INDICACIONES DE IMPLANTE DE TRONCO}

De forma general podemos afirmar que un IATC está indicado en aquellos casos de hipoacusia por lesión del nervio auditivo, los pacientes que con mayor frecuencia han sido incluidos en programas para la colocación de estos dispositivos han sido aquellos que padecían una Neurofibromatosis tipo 2 (NF2), que es un desorden autosómico dominante que afecta a 1 en 40.000 personas, en el que se desarrollan múltiples tumores en la médula espinal y en el cerebro. Su localización más común es el VIII par, siendo frecuentemente bilaterales. Generalmente comienzan en la porción vestibular, en su rama superior, y envuelven e invaden la porción auditiva Los pacientes se presentan con HSN progresiva, mala discriminación, tinitus, y en algunos casos vértigo y trastorno del equilibrio ${ }^{3,9}$.

Actualmente algunos centros han extendido las indicaciones del IATC. Elos plantean que podrían utilizarse en la mayoría de los casos en los que el implante coclear no es útil, ya sea por que el nervio coclear está muy comprometido, ausente o destruido como es el caso de la aplasia del nervio auditivo, la NF2, el neurinoma en oído único, la disrupción por trauma, la neuropatía auditiva o por defectos en la cóclea que hagan difícil, insatisfactorio o imposible la inserción del implante, como en la aplasia coclear, en las malformaciones severas como la cavidad común, y la osificación completa de la cóclea 5 .

\section{TÉCNICA QUIRÚRGICA}

日 conocimiento anatómico del núcleo coclear, del ángulo pontocerebeloso y en general de la base del cráneo son esenciales para el éxito de la implantación del IATC. $日$ complejo de los núcleos cocleares, compuesto por los núcleos ventral y dorsal, recibela información de las células ciliadas internas y externas del órgano de Corti, por la rama coclear del VIII par. Los núcleos cocleares se sitúan en la superficie del tronco cerebral cercanos a la salida del cuarto ventrículo, en la cara dorsolateral de éste. Esto crea un pequeño abultamiento visible en la superficie del tronco cerebral, que en la extensión tridimensional de los núcleos cocleares da sólo acceso a una pequeña área superficial, ya que la gran parte de los mismos queda oculta dentro del tronco ${ }^{12}$.

Las referencias anatómicas más importantes para localizar el complejo de los núcleos cocleares son la salida de los nervios craneanos VII , VIII y IX

Figura 2. Esquema de funcionamiento de un IATC.

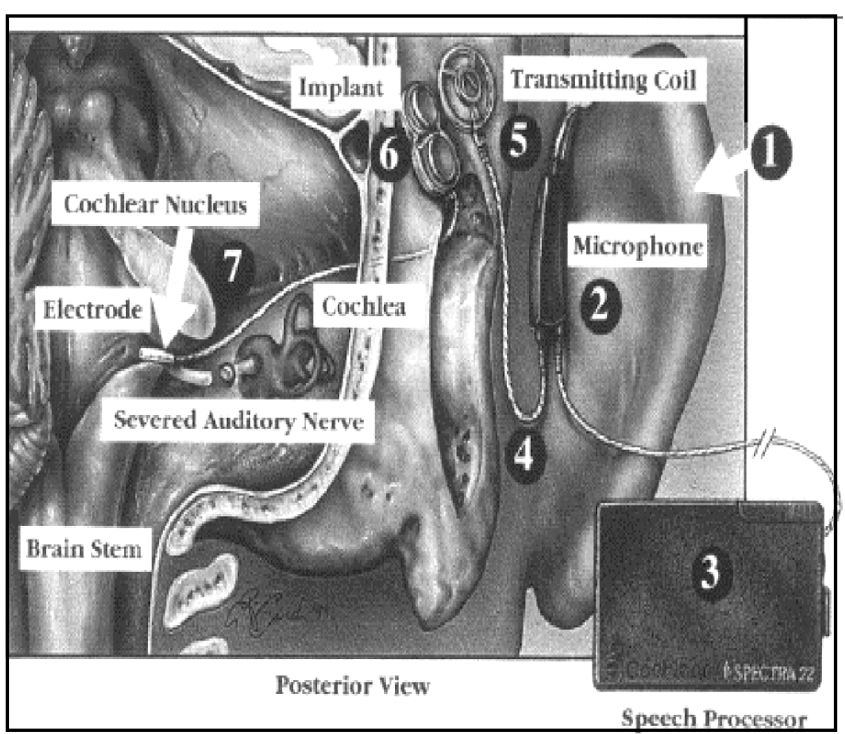


que forman un triángulo; el plexo coroideo, que protruye desde el foramen de Luschka el que provee el acceso al IV ventrículo y la prominencia del núcleo coclear en el IV ventrículo $0^{4,11,12}$.

Existen múltiples técnicas de abordaje, siendo las más utilizadas la suboccipital y la translaberíntica La decisión de seleccionar una vía de abordaje depende básicamente de la experiencia de cada centro ${ }^{4,11}$. Por lo general, la mayoría de los neurotólogos utilizan un abordaje translaberíntico, mientras que el suboccipital es el preferido por la mayor parte de los neurocirujanos ${ }^{12}$.

En el abordaje translaberíntico la incisión se realiza en la región retroauricular. Se fresa la región temporoparietal para la colocación del receptor. Para exponer las referencias anatómicas se debe retraer el flóculo cerebeloso, a continuación se procede a la identificación de la salida de los pares VI, VII, y IX'22. Los nervios $\mathrm{VI}$ y IX pueden ser estimulados y registra dos a través de un procedimiento electromiográfico3,12. Identificadas las referencias se procede a abrir la aracnoides y a entrar en el receso lateral del IV ventrículo tras lo cual se observa la salida de líquido céfalo raquídeo ${ }^{3,4}$. Una vez ubicados los núcleos se introduce el portaelectrodos del IATCen el receso lateral depositándolo sobrelasuperficiedelos núcleos cocleares. Deesta manerase estimula l complejo de los núcleos cocleares mayoritariamente el núcleo ventral ${ }^{12}$.

En laFigura3 se puede observar un esquemadela ubicación del portaelectrodos del IATCy unaimagen de su localización real en un espécimen correspondientea la necropsia de un paciente implantado. $\mathrm{\theta}$ núcleo ventral es el principal objetivo en la colocación de los electrodos ya que tiene entre sus características, ser el es más accesible, poser una gran densidad de neuronas, es el de mayor número de fibras ascendentes, el de menor número de sinapsis inhibitorias y la mejor tonotopía ${ }^{12}$.

Antes de finalizar las operaciones se debe verificar la adecuada colocación de los electrodos a través del registro de las respuestas eléctricas del tronco cerebral $^{12}$. Para esto se utilizan los Potenciales Evocados de troncoencéfalo (EABR) y la respuesta neural telemétrica (NRT) ${ }^{3}$. Los potenciales estimulados por $\mathrm{el}$ IATC consisten en tres curvas positivas, la primera corresponde a la onda III, la segunda a la IV y laúltima a la $V$ de un potencial normal. Los potenciales con latencias superiores a los 5 milisegundos son considerados no auditivos ${ }^{7}$. La optimización en la posición de los electrodos se realiza con el máximo número de electrodos con EABR positivos ${ }^{7}$. Ouando se obtienen EABR confiables el dispositivo de Dacron se dobla sobre el tronco para estabilizar el implante ${ }^{2-4}$. 日 receptor/estimulador es colocado en la zona de hueso temporal fresado. $\mathrm{\theta}$ imán interno de la antena y del receptor estimulador debe ser extraído y remplazado por un tapón de silicona para permitir la realización de futuros estudios con resonancia nuclear magnética ${ }^{2,4}$.

\section{PROGRAMACIÓN}

日 implante es activado entre 30 y 60 días posterior a la cirugía Se realiza en una Unidad de Cuidados Intensi-

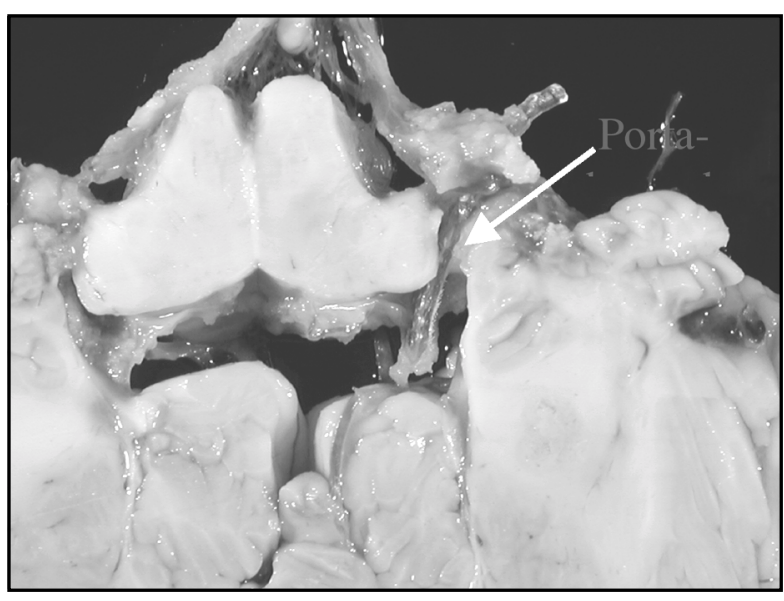

Figura 3. Necropsia de paciente implantado. 
vos con monitorización electrocardiográfica, por el riesgo de estimular estructuras del troncoencéfalo. $\theta$ desafío de la programación es encontrar aquellos electrodos que produzcan la mayor estimulación auditiva sin generar efectos colaterales 2,7 .

En un primer paso se configura el nivel máximo que es confortabley el mínimo que es percibido. Luego los canales que inducen sonidos no placenteros o que tienen efectos no auditivos como vértigo, estimulación del nervio facial y de las extremidades, entre otros, son excluidos. $\mathrm{日}$ problema se genera porque a diferencia de los implantes cocleares no existe una relación tonotópica definida entre el implante y el núcleo auditivo. I IATC es un electrodo de superficie mientras que la organización tonotópica del núcleo coclear es perpendicular, por lo que es difícil obtener una discrimina ción tonal con el implanter. En general se cumple la regla de que los electrodos más mediales y caudales producen tonos más agudos, mientras que los más cefálicos y laterales producen tonos más graves, pero esto es muy variable entre las personas especialmente en aquellos sujetos que no han recibido estimulación auditiva2. Por lo que se debe hacer una escala y un ranking para determinar el orden tonotópico apropiado de los electrodos. Esto se realiza estimulando dos electrodos secuencialmente e interrogando al paciente para determinar cuá es más agudo. Si 2 canales producen un tono similar uno es desconectado2,11. Esta información permite al técnico determinar la distribución tonotópica más adecuada para cada pa ciente. A finalizar las pruebas el micrófono externo es activado y según laimpresión del paciente el programa es nuevamente manipulado. Los pacientes son luego seguidos regularmente para evaluar la eficaciay seguridad de los implantes. Para esto se realizan varios test de percepción, entre ellos están las pruebas de detección de sonidos; la evaluación de lectura de labios con/ sin implante, con/sin visión; el reconocimiento de consonantes y vocales y el reconocimiento de oraciones cerradas y abiertas ${ }^{2-6}$.

\section{RESULTADOS}

En un estudio realizado por Lenarz en el Departamento de Otolarrinolaringología, de la Universidad de Hannover entre mayo 1996 y abril $2000^{7,8}$, se siguieron a 14 pacientes, con NF2 en los que se les colocó un IATC, por un promedio de 19 meses. Se observó que 12 de ellos recibieron sensación auditiva inmediatamente posterior a la activación del implante, en 1 paciente los electrodos migraron en el posoperatorio.

No se observaron complicaciones, sólo algunos pacientes presentaron respuestas no auditivas posterior a la activación como mareos y hormigueos. La lectura de labios de consonantes y vocales mejoró de $57 \%$ a $75 \%$ y de $78 \%$ a $92 \%$ respectivamente en 6 meses y aumentó la velocidad de lectura de labios de 15,3 palabras por minuto a 25,8 palabras por minuto. Para esta misma fecha la mitad de los pacientes fueron capaces de escuchar en algún grado consonantes y vocales sin lectura de labios.

Todos los pacientes fueron capaces de distinguir entre una conversación y un sonido ambiental, 2 de ellos fueron capaces de usar el teléfono de manera limitada y la mayoría consideró al implante bastante útil como ayuda para lectura de labios en ambientes silenciosos.

En otro estudio realizado por Kanowitz y cols entre julio 1994 y abril $2003^{3}$, con 18 pacientes con NF2 implantados, se realizó un seguimiento promedio de 21 meses en los que no se observaron complicaciones relacionadas directamente con el IATC y tampoco se presentaron problemas serios al estimular el tronco cerebral. Un paciente no obtuvo estimulación auditiva y en otro paciente luego de 6 meses la placa migró. Entre los efectos secundarios a la activación de implante se observaron movimientos faciales, sensaciones táctiles, movimientos contralaterales y desequilibrio. En el seguimiento se observó que 11 pacientes usaban el IATC por un promedio 9,6 horas al día; de los 7 no usuarios, 1 de ellos falleció, 3 obtenía mínimos beneficios, 1 implante no funcionó desde un comienzo, en otro los electrodos se desplazaron y otro el paciente dejó el seguimiento. Como resultados se observó que ningún paciente presentó discriminación en oraciones abiertas, que el IATC no proveía sonido de calidad normal, pero que permitía a los pacientes tener acceso a sonidos 
ambientales y mejorar su capacidad de comunicación.

En un estudio realizado por el House Ear Institute ${ }^{9}$, siguieron a 61 pacientes con NF2 que habían sido implantados con un IATC de 8 electrodos en el momento de la resección de los neurinomas. No se observaron complicaciones directamente atribuibles a la implantación de los electrodos y tampoco presentaron estímulos no auditivos serios. Como resultados se observó que 6 de los pacientes no presentaron estímulo auditivo, para la mayoría de los pacientes el beneficio radicó en una mejoría en la capacidad de lectura de labios y permitió la detección y reconocimiento de muchos sonidos ambientales. Los pacientes describían el sonido como si alguien hablara con la boca cerrada y la mayoría no fue capaz de reconocer palabras usando sólo la audición.

En otro estudio realizado por Vitorio Colleti y cols, entre abril 1997 y septiembre $2002^{2}$, incluyó a 29 pacientes tanto niños como adultos, con diversas patología que afectaban a la cóclea o al nervio coclear. Entre ellos habían 10 pacientes con NF2, 3 con neurinoma en oído único, 5 con aplasia coclear bilateral, 1 con neuropatía auditiva, 4 con osificación de la cóclea y 6 con traumatismo craneano. No se observaron complicaciones debido a la implantación y activación del IATC. Todos presentaron sensaciones auditivas posterior a la activación del implante y 20 de ellos tuvieron sensaciones no auditivas como mareos y hormigueo.

En laevaluación se realizaron pruebas en formato cerrado (se les entregaba por escrito ciertas palabras que luego tenían que repetir cuando se les eran nombradas) donde los pacientes sin tumor repitieron en promedio $56 \%$ de las palabras correctamentea los 6 meses y $86 \%$ a los 12 meses. En cambio los pacientes con tumor lo hicieron en $19 \%$ y $24 \%$ alos 6 y 12 meses, respectivamente. En pruebas de formato abierto, vale decir sin entregarles las palabras previa mente, al año de ser implantados los pacientes sin tumor respondían correctamenteen promedio el 63\% de las palabras, mientras que los pacientes con tumor lo hacían en promedio $12,2 \%$.

Como se puede observar existe una gran diferencia entre los resultados de los pacientes implantados por presentar tumor con los que se implantan por otra causa Los pacientes sin tumor tienen mejores resultados, lo que se puede explicar por que tienen una anatomía menos distorsionada, ya que la extracción de tumor y la cauterización de la zona pueden dañar al tronco; el mismo crecimiento y extracción del tumor puede dañar la irrigación del núcleo coclear, la expansión de núcleo coclear posterior al retiro de tumor puede modificar su relación con IATC y la compresión causada por el tumor puede provocar la degeneración de algunas neuronas del núcleo.

Existen múltiples factores que pueden ser los responsables del bajo rendimiento del implante en los pacientes con NF2, uno de ellos es la falta de estimulación selectiva, ya que el implante es colocado en la superficie del núcleo coclear, por lo que no se encuentra alineado tonotópicamente con éste. Además los electrodos están muy alejados de las neuronas, lo que incrementa sus niveles de activación y por ende la información se dispersa reduciendo su selectividad. Esta degradación de la resolución reduce la habilidad del receptor de lograr una discriminación de las frecuencias, lo que ha demostrado ser esencial en la percepción del lenguaje. Por esto se desarrolló un IATC penetrante, el cual incorpora microelectrodos tipo aguja de diferentes longitudes, que penetran en el tronco encefálico, y estimulan selectivamente las distintas regiones tonotópicas del núcleo. Se espera que con una mayor cercanía a las neuronas se logre un acceso más selectivo a los distintos tonos, y que por ende se obtengan mejores resultados, similares a los obtenidos con los implantes cocleares multicanales $^{13}$.

\section{CONCLUSIONES}

En base a la información recopilada y presentada en esta revisión, podemos apreciar que es necesario un equipo multidisciplinario para la realización de un implante; que además se requiere de una colaboración persistente y paciente por parte de los usuarios y del equipo tratante; que estos implantes son seguros, ya que no aumentan la morbilidad 
operatoria y no producen secuelas neurológicas y que los resultados son variados desde sólo la percepción de sonidos ambientales, similar a los resultados obtenidos con los implantes cocleares monocanales, hasta la comunicación por teléfono, aunque la mayoría de las publicaciones concuerdan que la meta principal, en los pacientes con NF2, es ayudarlos a percibir los sonidos ambientales y a fortalecer la habilidad de lectura labial.

De lo único que podemos estar seguros es que éste continúa siendo un campo emergente, en el que se están desarrollando nuevas tecnologías como los implantes penetrantes y en el que se está ampliando su uso a pacientes con diversas patologías.

\section{BIBLIOGRAFÍA}

1. EMURUGASU. Recent Advances in the Treatment of Sensorioneural Deafness. Ann Acad Med Singapore 2005; 34: 313-21.

2. Vittorio Colletti, maroo Carner, Veronica MiORELLI ET AL. Auditory Brainstem Implant $(\mathrm{ABI})$ : New Frontiers in Adults and Children. Otolaryngology-Head and Neck Surgery 2005; 133:126-38.

3. KanOWTIZ SETH J, ShapiRo William H, GOLFnOS JOHN G ET AL. Auditory Brainstem Implantation in Patients with Neurofibromatosis Type 2. Laryngoscope 2005; 114 (12): 2135-46.

4. VitTORIO COLletti, FrancesCo Horino, MarCO CARNER ET AL. Auditory Brainstem Implantation: The University of Verona Experience. Otolaryngology-Head and Neck Surgery 2002; 127: 8496.

5. Vittorio colletti, marco Carner, francesco FoRINO ET AL. Hearing Restoration with Auditory Brainstem Implant in Three Children with Cochlear Nerve Aplasia. Oology and Neurotology 2002, 23 (5):682-93.
6. DAVID R FRIEDLAND, PHILIP A WACKMM. Evaluation of Surgical Approaches to Endoscopic Auditory Brainstem Implantation. Laryngoscope 1999; 109: 175-80.

7. Lenarz ThOMAS, MOSHR日 MinOO, MatTHES CORDULA et AL. Auditory Brainstem Implant Part I : Auditory Performance and Its Evolution Over Time. Aology and Neurotology 2001, 22 (6): 823-33.

8. Lenarz ThOMas, Moshreg Minoo, Matthies CORDULA ET AL. Auditory Brainstem Implant Part II: Subjective Assessment of Functional Outcome. Otology and Neurotology 2002, 23 (5): 694-7.

9. Steven R Otto, ERALd E Brackmann, William E HITSE BERGER, ROBERT B SHANNON, JOHANNES KUCHTA. Multichannel Auditory Brainstem Implant: update on performance in 61 patients. $J$ Neurosurgery 2002, 96: 1063-71.

10. COllett VitTORIO, horino FrancesoO G, CarNeR MAROO, MIORELII VERONICA, GIIDA MAURIZIO, COLleTTI LILIANA. Auditory Brainstem Implant as a Salvage Treatment alter Unsuccessful Cochlear Implantation. Otology and Neurotology 2004; 25 (4): 485-96.

11. COlletti Vittorio, horino francesco G, CarNER MARCO ET AL. The Retrosigmoid Approach for Auditory Brainstem Implantation. Am J Otol 2000; 21: 826-36.

12. Manue ManRIQUe, EMILIO García-IBAÑE, LUIS García-IBAÑEZ, ANGe Ramos. Técnica Quirúrgica para la Colocación del Implante Auditivo de Tronco Cerebral. XVII Congreso Nacional de la Sociedad Española de Otorrinolaringología y Patología Cérvico-Facial 1998.

13. Thomas lenarz, hubert h lim, Guenter ReuTER, JAMES F PATRICK, MinOo Lenarz. The Auditory Midbrain Implant: A New Auditory Prosthesis for Neural Deafness-Concept and Device Description. Otology and Neurotology 2006; 27 (6): 838-43. 